\title{
On graphs with no induced five-vertex path or paraglider
}

\author{
Shenwei Huang* \\ T. Karthick ${ }^{\dagger}$
}

March 28, 2019

\begin{abstract}
Given two graphs $H_{1}$ and $H_{2}$, a graph is $\left(H_{1}, H_{2}\right)$-free if it contains no induced subgraph isomorphic to $H_{1}$ or $H_{2}$. For a positive integer $t$, $P_{t}$ is the chordless path on $t$ vertices. A paraglider is the graph that consists of a chorless cycle $C_{4}$ plus a vertex adjacent to three vertices of the $C_{4}$. In this paper, we study the structure of ( $P_{5}$, paraglider $)$-free graphs, and show that every such graph $G$ satisfies $\chi(G) \leq\left\lceil\frac{3}{2} \omega(G)\right\rceil$, where $\chi(G)$ and $\omega(G)$ are the chromatic number and clique number of $G$, respectively. Our bound is attained by the complement of the Clebsch graph on 16 vertices. More strongly, we completely characterize all the $\left(P_{5}\right.$, paraglider)-free graphs $G$ that satisfies $\chi(G)>\frac{3}{2} \omega(G)$. We also construct an infinite family of ( $P_{5}$, paraglider)-free graphs such that every graph $G$ in the family has $\chi(G)=\left\lceil\frac{3}{2} \omega(G)\right\rceil-1$. This shows that our upper bound is optimal up to an additive constant and that there is no $\left(\frac{3}{2}-\epsilon\right)$ approximation algorithm to the chromatic number of ( $P_{5}$, paraglider)-free graphs for any $\epsilon>0$.
\end{abstract}

Keywords. $P_{5}$-free graphs; Chromatic number; Clique number.

\section{Introduction}

Graphs in this paper are simple and finite. Given a positive integer $\ell$, we denote the path on $\ell$ vertices by $P_{\ell}$, and we denote the complete graph on $\ell$ vertices by $K_{\ell}$. For an integer $\ell \geq 3, C_{\ell}$ is the cycle on $\ell$ vertices. A paraglider is the graph that consists of a $C_{4}$ plus a vertex adjacent to three vertices of the $C_{4}$. Given two graphs $G$ and $H$, we denote by $G \cup H$ the disjoint union of $G$ and $H$, and by $G+H$ the join of $G$ and $H$. The union of $k$ copies of the same graph $G$ will be denoted by $k G$; for example $2 K_{2}$ denotes the graph that consists in two disjoint copies of $K_{2}$. The complement of a graph $G$ is denoted by $\bar{G}$. A hole (antihole) in a graph is an induced subgraph that is isomorphic to $C_{\ell}\left(\overline{C_{\ell}}\right)$ with $\ell \geq 4$, and $\ell$ is the length of the hole (antihole). A hole or an antihole is odd if $\ell$ is odd. Given a family of graphs $\mathcal{H}$, a graph $G$ is $\mathcal{F}$-free if no induced

\footnotetext{
${ }^{*}$ College of Computer Science, Nankai University, Tianjin 300350, China. This research is partially supported by the National Natural Science Foundation of China (11801284).

${ }^{\dagger}$ Computer Science Unit, Indian Statistical Institute, Chennai Centre, Chennai 600029, India. This research is partially supported by SERB-DST, Government of India under MATRICS scheme.
} 
subgraph of $G$ is isomorphic to a member of $\mathcal{F}$; when $\mathcal{F}$ has only one element $H$ we say that $G$ is $H$-free; when $\mathcal{F}$ has two elements $H_{1}$ and $H_{2}$, we simply write $G$ is $\left(H_{1}, H_{2}\right)$-free instead of $\left\{H_{1}, H_{2}\right\}$-free.

For any integer $k$, a $k$-coloring of a graph $G$ is a mapping $\psi: V(G) \rightarrow$ $\{1, \ldots, k\}$ such that $\psi(u) \neq \psi(v)$ whenever $u$ and $v$ are adjacent in $G$. A graph is $k$-colorable if it admits a $k$-coloring. The chromatic number $\chi(G)$ of a graph $G$ is the smallest integer $k$ such that $G$ is $k$-colorable. A clique in a graph $G$ is a set of pairwise adjacent vertices, and the clique number of $G$, denoted by $\omega(G)$, is the size of a maximum clique in $G$. Obviously $\chi(H) \geq \omega(H)$ for every induced subgraph $H$ of $G$. A graph $G$ is perfect if every induced subgraph $H$ of $G$ satisfies $\chi(H)=\omega(H)$. Chudnovsky et al. 8] showed that a graph is perfect if and only if it does not contain an odd hole or an odd antihole as an induced subgraph, and is known as the Strong Perfect Graph Theorem (SPGT). A class of graphs $\mathcal{G}$ is said to be $\chi$-bounded 16 if there is a function $f$ (called a $\chi$-binding function) such that every $G \in \mathcal{G}$ satisfies $\chi(G) \leq f(\omega(G))$. For instance, the class of perfect graphs is $\chi$-bounded with identity function $f(x)=x$ as the $\chi$ binding function. In fact, several classes of graphs are known to be $\chi$-bounded; see [12, 14, 17, 18.

Gyárfás [16 studied the $\chi$-boundedness for the class of $P_{t}$-free graphs, and showed that every $P_{t}$-free graph $G$ has $\chi(G) \leq(t-1)^{\omega(G)-1}$. It is well known that for $t \leq 4, P_{t}$-free graphs are perfect. The problem of determining whether the class of $P_{t}$-free graphs $(t \geq 5)$ admits a polynomial $\chi$-binding function remains open, and seems to be difficult even when $t=5$. Moreover, the existence of polynomial $\chi$-binding function for the class of $P_{t}$-free graphs $(t \geq 5)$ would imply the Erdös-Hajnal conjecture for $P_{t}$-free graphs; see [5]. The best known $\chi$-binding function $f$ for the class of $P_{5}$-free graphs satisfies $c\left(\omega^{2} / \log w\right) \leq$ $f(\omega) \leq 2^{\omega}$; see [13. Here we are interested in $\chi$-binding functions for the class of $\left(P_{5}, H\right)$-free graphs, for various graphs $H$. Recently, Brause et al. 2] showed that the class of $\left(2 K_{2}, 3 K_{1}\right)$-free graphs does not admit a linear $\chi$ binding function. It follows that the class of $\left(P_{5}, H\right)$-free graphs, where $H$ is any $P_{5}$-free graph with independence number $\alpha(H) \geq 3$, does not admit a linear $\chi$-binding function. Thus it is interesting to the study of $\chi$-boundedness for the class of $\left(P_{5}, H\right)$-free graphs where $\alpha(H) \leq 2$. Choudum et al. [3] showed that every $\left(P_{5}, C_{4}\right)$-free graph $G$ satisfies $\chi(G) \leq\left\lceil\frac{5 \omega(G)}{4}\right\rceil$, and that every $\left(P_{5}, K_{1}+\right.$ $C_{4}$ )-free graph $G$ satisfies $\chi(G) \leq 5\left\lceil\frac{5 \omega(G)}{4}\right\rceil$. It is shown in [11, 18, that every ( $P_{5}$, diamond)-free graph $G$ satisfies $\chi(G) \leq \omega(G)+1$, and in [2] that every $\left(P_{5}\right.$, paw)-free graph $G$ satisfies $\chi(G) \leq \omega(G)+1$. Chudnovsky and Sivaram 77 showed that every $\left(P_{5}, C_{5}\right)$-free graph $G$ satisfies $\chi(G) \leq 2^{\omega(G)-1}$. Fouquet et al. 99 proved that there are infinitely many $\left(P_{5}, \overline{P_{5}}\right)$-free graphs $G$ with $\chi(G) \geq \omega(G)^{\mu}$, where $\mu=\log _{2} 5-1$, and that every $\left(P_{5}, \overline{P_{5}}\right)$-free graph $G$ satisfies $\chi(G) \leq\left(\begin{array}{c}\omega(G)+1 \\ 2\end{array}\right)$. Very recently, Chudnovsky et al. [6] showed that every $\left(P_{5}, K_{1}+P_{4}\right)$-free graph $G$ satisfies $\chi(G) \leq\left\lceil\frac{5 \omega(G)}{4}\right\rceil$. We refer to a recent comprehensive survey of Schiermeyer and Randerath [18] for more results.

In this paper, we study the structure of the class of $\left(P_{5}\right.$, paraglider $)$-free graphs, and show that every such graph $G$ satisfies $\chi(G) \leq\left\lceil\frac{3 \omega(G)}{2}\right\rceil$. Our bound is attained by the complement of the well-known 5-regular Clebsch graph on 16 vertices. More strongly, we completely characterize all the $\left(P_{5}\right.$, paraglider $)$-free graphs $G$ that satisfies $\chi(G)>\frac{3}{2} \omega(G)$. We also construct an infinite family of $\left(P_{5}\right.$, paraglider)-free graphs such that every graph $G$ in the family has $\chi(G)=$ 
$\left\lceil\frac{3}{2} \omega(G)\right\rceil-1$. This shows that our upper bound is optimal up to an additive constant, and that there is no $\left(\frac{3}{2}-\epsilon\right)$-approximation algorithm to the chromatic number of $\left(P_{5}\right.$, paraglider $)$-free graphs for any $\epsilon>0$. Moreover, our results generalizes the results known on the existence of linear $\chi$-binding functions for $\left(P_{5}, C_{4}\right)$-free graphs, $\left(P_{5}\right.$, paw)-free graphs, $\left(P_{5}\right.$, diamond $)$-free graphs, and for $\left(3 K_{1}\right.$, paraglider)-free graphs [4].

\section{Notations and Preliminaries}

We use standard notation and terminology. In a graph $G$, the neighborhood of a vertex $x$ is the set $N_{G}(x)=\{y \in V(G) \backslash\{x\} \mid x y \in E(G)\}$; we drop the subscript $G$ when there is no ambiguity. The non-neighborhood of a vertex $x$ is the set $V(G) \backslash(N(x) \cup\{x\})$, and is denoted by $\overline{N(x)}$. A vertex is universal if it is adjacent to all other vertices. Two non-adjacent vertices $u$ and $v$ in a graph $G$ are comparable if $N(u) \subseteq N(v)$ or $N(v) \subseteq N(u)$. For any $x \in V(G)$ and $A \subseteq V(G) \backslash x$, we let $N_{A}(x)=N(x) \cap A$. Let $X$ be a subset of $V(G)$. We denote by $G[X]$ the subgraph induced by $X$ in $G$. For simplicity, we write $G \backslash X$ instead of $G[V(G) \backslash X]$. Further if $X$ is singleton, say $\{v\}$, we write $G-v$ instead of $G \backslash\{v\}$. For any two subsets $X$ and $Y$ of $V(G)$, we denote by $[X, Y]$, the set of edges that has one end in $X$ and other end in $Y$. We say that $X$ is complete to $Y$ or $[X, Y]$ is complete if every vertex in $X$ is adjacent to every vertex in $Y$; and $X$ is anticomplete to $Y$ if $[X, Y]=\emptyset$. If $X$ is singleton, say $\{v\}$, we simply write $v$ is complete (anticomplete) to $Y$ instead of writing $\{v\}$ is complete (anticomplete) to $Y$. We say that a subgraph $H$ of $G$ is dominating if every vertex in $V(G) \backslash V(H)$ is a adjacent to a vertex in $H$. A clique-cutset of a graph $G$ is a clique $K$ in $G$ such that $G \backslash K$ has more connected components than $G$. An atom is a connected graph without a clique-cutset.

A stable set is a set of pairwise non-adjacent vertices. We say that two sets meet if their intersection is not empty. In a graph $G$, we say that a stable set is good if it meets every clique of size $\omega(G)$.

An expansion of a graph $H$ is any graph $G$ such that $V(G)$ can be partitioned into $|V(H)|$ non-empty sets $Q_{v}, v \in V(H)$, such that $\left[Q_{u}, Q_{v}\right]$ is complete if $u v \in E(H)$, and $\left[Q_{u}, Q_{v}\right]=\emptyset$ if $u v \notin E(H)$. An expansion of a graph is a clique expansion if each $Q_{v}$ is a clique, is a $\overline{P_{3}}$-free expansion if each $Q_{v}$ induces a $\overline{P_{3}}$-free graph, and is a perfect expansion if each $Q_{v}$ induces a perfect graph. By a classical result of Lovász [15], any perfect expansion of a perfect graph is perfect. In particular, any $\overline{P_{3}}$-free expansion of a perfect graph is perfect.

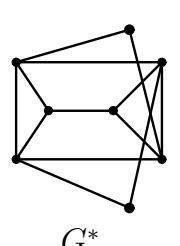

$G^{*}$

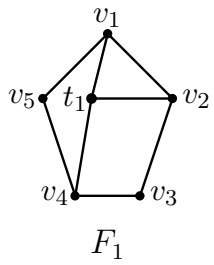

$F_{1}$

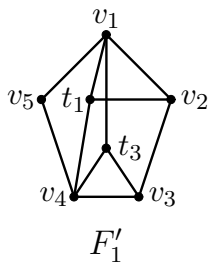

$F_{1}^{\prime}$

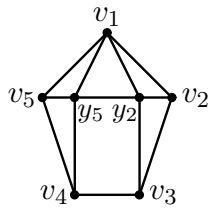

$F_{2}$

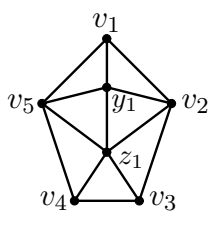

$F_{3}$

Figure 1: Some special graphs

Let $G^{*}, F_{1}, F_{1}^{\prime}, F_{2}, F_{3}$ be five graphs as shown in Figure 1. 
Let $\mathcal{H}$ be the class of graphs $G$ such that $V(G)$ can be partitioned into five sets $Q_{1}, Q_{2}, R_{1}, R_{2}, S$ such that:

- $Q_{1}=\left\{a_{1}, a_{2} \ldots, a_{k}\right\}, Q_{2}=\left\{b_{1}, b_{2}, \ldots, b_{k}\right\}$ (where $k \geq 2$ ), $S$ are cliques, $\left[Q_{1}, Q_{2}\right]$ is a perfect matching, say $\left\{a_{1} b_{1}, a_{2} b_{2}, \ldots, a_{k} b_{k}\right\}$ and $|S| \leq k$.

- $G\left[R_{1}\right]$ and $G\left[R_{2}\right]$ are perfect.

- $\left[Q_{1}, R_{1}\right],\left[Q_{2}, R_{2}\right]$ are complete, $\left[Q_{1} \cup R_{1}, R_{2}\right]=\emptyset$ and $\left[Q_{2} \cup R_{2}, R_{1}\right]=\emptyset$.

- $\left[S, R_{1} \cup R_{2}\right]$ is complete.

- There exists an injective function $f: S \rightarrow\{1,2, \ldots, k\}$ such that for each vertex $x \in S,\{x\}$ is anti-complete to $\left\{a_{f(x)}, b_{f(x)}\right\}$, and is complete to $\left(Q_{1} \cup Q_{2}\right) \backslash\left\{a_{f(x)}, b_{f(x)}\right\}$.

- No other edges in $G$.

Clearly, the graphs $\overline{C_{6}}$ and $F_{2}$ belong to $\mathcal{H}$. See Section 4 for more examples.

We will use the following theorem of Brandstädt and Hoàng [1].

Theorem 1 ([1]) Let $G$ be a ( $P_{5}$, paraglider)-free atom that has no universal or pair of comparable vertices. Then either $G$ is $G^{*}$ or every induced $C_{5}$ in $G$ is dominating.

\section{Structure of $\left(P_{5}\right.$, paraglider $)$-free graphs}

In this section, we prove the following structure theorem for the class of $\left(P_{5}\right.$, paraglider)-free graphs.

Theorem 2 Let $G$ be a $\left(P_{5}\right.$, paraglider)-free atom with no universal or pair of comparable vertices. Then one of the following hold:

- $G$ is an induced subgraph of the complement of the Clebsch graph.

- $G$ is a $\overline{P_{3}}$-free expansion of $C_{5}$.

- $G$ has a stable set $S$ such that either $S$ is good or $G \backslash S$ is perfect.

- $G \in \mathcal{H}$.

Proof. If $G$ is $G^{*}$, then $S:=\left\{v_{7}, v_{8}\right\}$ is a stable set such that $G \backslash S \cong \overline{C_{6}}$ is perfect. If $G$ is perfect, then any color class in a $\chi(G)$-coloring of $G$ is a good stable set. So we may assume that $G$ is not $G^{*}$, and is not perfect. Now since a $P_{5}$-free graph contains no hole of length at least 7 , and a paraglider-free graph contains no antihole of length at least 7 , it follows by the Strong Perfect Graph Theorem 8 that $G$ contains a hole of length 5 . That is, $G$ contains a $C_{5}$ as an induced subgraph. Now the theorem follows from Theorem 1 , and from Theorems 4 5, 6and Theorem 7 given below.

In the next theorem, we make some general observations about the situation when a $\left(P_{5}\right.$, paraglider)-free graph contains a hole (which must have length 5$)$. 
Theorem 3 Let $G$ be any ( $P_{5}$, paraglider)-free graph that contains a $C_{5}$ with vertex-set $C=\left\{v_{1}, \ldots, v_{5}\right\}$ and $\left\{v_{i} v_{i+1} \mid i \in\{1, \ldots, 5\}, i \bmod 5\right\}$. Suppose that $G$ is an atom and has no pair of comparable vertices. Let:

$$
\begin{aligned}
T_{i} & =\left\{x \in V(G) \backslash C \mid N_{C}(x)=\left\{v_{i}, v_{i+1}, v_{i+3}\right\}\right\} . \\
X_{i} & =\left\{x \in V(G) \backslash C \mid N_{C}(x)=\left\{v_{i-1}, v_{i+1}\right\}\right\} . \\
Y_{i} & =\left\{x \in V(G) \backslash C \mid N_{C}(x)=\left\{v_{i-1}, v_{i}, v_{i+1}\right\}\right\} . \\
Z_{i} & =\left\{x \in V(G) \backslash C \mid N_{C}(x)=C \backslash\left\{v_{i}\right\}\right\} . \\
A & =\left\{x \in V(G) \backslash C \mid N_{C}(x)=C\right\} .
\end{aligned}
$$

Moreover, let $T=T_{1} \cup \cdots \cup T_{5}, X=X_{1} \cup \cdots \cup X_{5}, Y=Y_{1} \cup \cdots \cup Y_{5}$, and $Z=Z_{1} \cup \cdots \cup Z_{5}$. Then the following properties hold for all $i$, $i \bmod 5$ :

(R1) $G[C]$ is a dominating induced subgraph of $G$ and $V(G)=C \cup A \cup T \cup X \cup$ $Y \cup Z$.

(R2) (a) $\left|T_{i}\right| \leq 1$. If $\left|T_{i}\right|=1$, then we denote $T_{i}$ by $\left\{t_{i}\right\}$.

(b) $\left[T_{i}, T_{j}\right]=\emptyset$, for every $j$; so $T$ is an independent set.

(c) $\left[T_{i}, X_{i+3}\right]$ is complete, and $\left[T_{i}, X_{j}\right]=\emptyset$, for every $j \neq i+3$.

(R3) (a) $X_{i}$ is an independent set.

(b) $\left[X_{i}, X_{i+1}\right]$ is complete.

(c) $\left|\left[X_{i}, X_{i+2}\right]\right| \leq 1$.

(d) If $X_{i+1} \neq \emptyset$, then $\left[X_{i}, X_{i+2}\right]=\emptyset$.

(R4) (a) $G\left[Y_{i}\right]$ is $\left(K_{2} \cup K_{1}\right)$-free. Hence $G\left[Y_{i}\right]$ is a complete multi-partite graph.

(b) $\left[Y_{i}, Y_{i+1}\right]$ is complete.

(c) If $\left[Y_{i}, Y_{i+2}\right] \neq \emptyset$, then $\left[Y_{i}, Y_{i+2}\right]$ is a matching.

(d) If $Y_{i} \neq \emptyset$, then $Y_{i-1}$ and $Y_{i+1}$ are cliques.

(e) If $y \in Y_{i}$ and if $\left[\{y\}, Y_{i+2}\right]$ is complete, then $\left|Y_{i+2}\right| \leq 1$. More generally, if $\left[Y_{i}, Y_{i+2}\right]$ is complete, then $\left|Y_{i}\right| \leq 1$ and $\left|Y_{i+2}\right| \leq 1$.

(R5) (a) $\left|Z_{i}\right| \leq 1$. If $\left|Z_{i}\right|=1$, then we denote $Z_{i}$ by $\left\{z_{i}\right\}$.

(b) $\left[Z_{i}, Z_{i+1}\right]=\emptyset$.

(c) $\left[Z_{i}, Z_{i+2}\right]$ is complete.

(R6) (a) $\left[X_{i}, Y_{i} \cup Y_{i+1} \cup Y_{i-1} \cup\left(Z \backslash Z_{i}\right)\right]$ is complete.

(b) $\left[X_{i}, Y_{i-2} \cup Y_{i+2} \cup Z_{i}\right]=\emptyset$.

(R7) (a) $\left[Z_{i}, Y_{i} \cup Y_{i-2} \cup Y_{i+2}\right]$ is complete.

(b) $\left[Z_{i}, Y_{i-1} \cup Y_{i+1}\right]=\emptyset$.

(R8) (a) $A$ is a clique.

(b) $[A, V(G) \backslash(A \cup Y)]$ is complete.

(R9) Suppose that $T_{i} \neq \emptyset$. Then:

(a) The sets $T_{i-1} \cup T_{i+1}, Y, Z \backslash Z_{i+3}$ are empty.

(b) $\left[X_{i}, X_{i+2}\right],\left[X_{i+1}, X_{i-1}\right]$ and $\left[T_{i}, Z_{i+3}\right]$ are empty.

(R10) Let $x, y \in V(G)$ and $q \in A$. Then the following hold:

(a) If $x \in Y_{i}$ and $y \in Y_{i+1} \cup Y_{i+2}$ are adjacent, then $q$ is either complete or anti-complete to $\{x, y\}$.

(b) If $x \in Y_{i}$ and $y \in Y_{i+2}$ are not adjacent, then $q$ is adjacent one of $x$, y.

(c) If $x \in Y_{i}$ and $y \in Y_{i}$ are adjacent, then $q$ is adjacent to one of $x, y$.

(d) $\overline{N(q)} \cap Y_{i}$ is a stable set. 
(R11) Suppose that $G$ is $F_{1}$-free. Then $\left[X_{i}, X_{i+2} \cup X_{i-2} \cup Y_{i+2} \cup Y_{i-2}\right]=\emptyset$, and hence $X=\emptyset$.

Proof. Let $G$ be the given graph with vertex-set $V$ and edge-set $E$.

Proof of (R I . Since $G$ has no clique cut-set, by Theorem 1 $G[C]$ is dominating, and so every vertex in $V \backslash C$ has a neighbor in $C$. Now (R/1) follows since $G$ is $P_{5}$-free. Indeed if a vertex $x \in V \backslash C$ has exactly one neighbor (say, $v_{i}$ ) or has exactly two neighbors that are consecutive (say, $v_{i}$ and $v_{i+1}$ ) in $C$, then $x-v_{i}-v_{i-1}-v_{i-2}-v_{i-3}$ is a $P_{5}$.

Proof of (R2) ( (a): Otherwise, for any two vertices $x$ and $y$ in $T_{i}$, either $\left\{x, v_{i+1}\right.$, $\left.v_{i+2}, v_{i+3}, y\right\}$ or $\left\{x, v_{i}, y, v_{i+3}, v_{i+1}\right\}$ induces a paraglider. So (a) holds.

(b): Suppose to the contrary that there are adjacent vertices $x \in T_{i}$ and $y \in T_{j}$. Now if $j \neq i-1$, then $\left\{x, v_{i+1}, v_{i+2}, v_{i+3}, y\right\}$ induces a paraglider, and if $j=i-1$, then $\left\{y, v_{i}, v_{i+1}, v_{i+2}, x\right\}$ induces a paraglider, a contradiction. This proves item $(b)$.

(c): Pick a vertex $x \in T_{i}$ and a vertex $y \in X_{j}$. Up to symmetry, we may assume that $j \in\{i, i+2, i+3\}$. If $j=i$, then $x y \notin E$, for otherwise $\left\{y, v_{i+1}, v_{i}, v_{i-1}, x\right\}$ induces a paraglider. If $j=i+2$, then $x y \notin E$, for otherwise $\left\{x, v_{i+1}, v_{i+2}, v_{i+3}, y\right\}$ induces a paraglider. If $j=i+3$, then $x y \in E$, for otherwise $v_{i}-x-v_{i+3}-v_{i+2}-y$ is a $P_{5}$. Since this holds for any $x$ and $y$, it proves item (c).

Proof of (R3). (a): Otherwise, for any two adjacent vertices $x$ and $y$ in $X_{i}$, $\left\{x, v_{i+1}, v_{i}, v_{i-1}, y\right\}$ induces a paraglider. So (a) holds.

(b): Suppose not, and let $x \in X_{i}$ and $y \in X_{i+1}$ be not adjacent. Then $x-v_{i-1}$ $v_{i-2}-v_{i-3}-y$ is a $P_{5}$. So (b) holds.

$(c)$ : We may assume that $\left[X_{i}, X_{i+2}\right] \neq \emptyset$. We first claim that $\left[X_{i}, X_{i+2}\right]$ is a matching. Suppose not. Then, up to symmetry, we may assume that there exist vertices $x \in X_{i}$ and $y, z \in X_{i+2}$ such that $x y, x z \in E$. By (a), $y z \notin E$. But then $\left\{x, z, v_{i+3}, y, v_{i+1}\right\}$ induces a paraglider. So $\left[X_{i}, X_{i+2}\right]$ is a matching. Now, if $\left|\left[X_{i}, X_{i+2}\right]\right| \geq 2$, then there exist matching edges $e, f \in\left[X_{i}, X_{i+2}\right]$, say $e:=x y$ and $f:=x^{\prime} y^{\prime}$ with $x, x^{\prime} \in X_{i}$ and $y, y^{\prime} \in X_{i+2}$. By (a), we have $x x^{\prime} \notin E$ and $y y^{\prime} \notin E$. But then $y-x-v_{i-1}-x^{\prime}-y^{\prime}$ is a $P_{5}$, a contradiction. This proves item (c).

Proof of (R4). (a): Suppose to the contrary that $G\left[Y_{i}\right]$ contains an induced $K_{2} \cup K_{1}$ with vertex-set $\{x, y, z\}$ and edge-set $\{x y\}$. Then $\left\{x, v_{i-1}, z, v_{i+1}, y\right\}$ induces a paraglider, which is a contradiction. So (a) holds.

(b): Suppose not, and let $x \in Y_{i}$ and $y \in Y_{i+1}$ be not adjacent. Then $x-v_{i-1^{-}}$ $v_{i-2}-v_{i-3}-y$ is a $P_{5}$. So (b) holds.

(c): Suppose not. We may assume, up to symmetry, that $x \in Y_{i}$ and $y, z \in Y_{i+2}$ such that $x y, x z \in E$. Then $\left\{x, y, v_{i-2}, v_{i-1}, z\right\}$ or $\left\{x, y, v_{i-2}, z, v_{i+1}\right\}$ induces a paraglider, a contradiction. This proves item (c).

$(d)$ : Let $x \in Y_{i}$. Suppose to the contrary that there are non-adjacent vertices $y$ and $z$ in $Y_{i+1}$. By (b), $x y, y z \in E$. But then $\left\{x, y, v_{i+2}, z, v_{i}\right\}$ induces a paraglider which is a contradiction. So $Y_{i+1}$ is a clique. Likewise, $Y_{i-1}$ is a clique. This proves item (d).

(e): This follows by item (c).

Proof of (R 5 . (a): Otherwise, for any two vertices $x$ and $y$ in $Z_{i}$, either $\left\{v_{i}, v_{i+1}\right.$, $\left.x, v_{i-1}, y\right\}$ or $\left\{x, v_{i+1}, y, v_{i-1}, v_{i+2}\right\}$ induces a paraglider. 
(b): Suppose not, and let $x \in Z_{i}$ and $y \in Z_{i+1}$ be adjacent. Then $\left\{v_{i}, v_{i+1}\right.$, $\left.v_{i+2}, y, x\right\}$ induces a paraglider.

$(c)$ : Suppose not, and let $x \in Z_{i}$ and $y \in Z_{i+2}$ be not adjacent. Then $\left\{v_{i}, v_{i+1}\right.$, $\left.x, v_{i-1}, y\right\}$ induces a paraglider.

Proof of (R[6). (a): Suppose not, and let $x \in X_{i}$ and $y \in Y_{i} \cup Y_{i+1} \cup Y_{i-1} \cup\left(Z \backslash Z_{i}\right)$ be non-adjacent. By symmetry, we may assume that $y \in Y_{i} \cup Y_{i+1} \cup Z_{i-1} \cup Z_{i+2}$. Now if $y \in Y_{i} \cup Z_{i+2}$, then $\left\{x, v_{i+1}, y, v_{i-1}, v_{i}\right\}$ induces a paraglider, and if $y \in Y_{i+1} \cup Z_{i-1}$, then $x-v_{i-1}-v_{i}-y-v_{i+2}$ is a $P_{5}$, a contradiction. This proves item (a).

(b): Suppose not, and let $x \in X_{i}$ and $y \in Y_{i-2} \cup Y_{i+2} \cup Z_{i}$ be adjacent. By symmetry, we may assume that $y \in Y_{i+2} \cup Z_{i}$. Now if $y \in Y_{i+2}$, then $v_{i}-v_{i-1^{-}}$ $x-y-v_{i+2}$ is a $P_{5}$, and if $y \in Z_{i}$, then $\left\{x, v_{i+1}, v_{i}, v_{i-1}, y\right\}$ induces a paraglider, a contradiction. This proves item (b).

Proof of (R/7). (a): Suppose not. Up to symmetry, we may assume that there are non-adjacent vertices $x \in Z_{i}$ and $y \in Y_{i} \cup Y_{i+2}$. Now if $y \in Y_{i}$, then $\left\{v_{i}, v_{i+1}, x, v_{i-1}, y\right\}$ induces a paraglider, and if $y \in Y_{i+2}$, then $v_{i}-v_{i-1}-x-v_{i+2}-y$ is a $P_{5}$, a contradiction. This proves item (a).

(b): Suppose not, and let $x \in Z_{i}$ and $y \in Y_{i+1} \cup Y_{i-1}$ be adjacent. Then $\left\{v_{i}, v_{i+1}, x, v_{i-1}, y\right\}$ induces a paraglider.

Proof of (R8). Suppose not, and let $x \in A$ and $y \in A \cup(V(G) \backslash Y)$ be nonadjacent. If $y \in A$, then $\left\{x, v_{i}, y, v_{i+3}, v_{i+1}\right\}$ induces a paraglider. So let us assume that $y \in V(G) \backslash Y$. Then there exist $j \in\{1, \ldots, 5\}, j$ modulo 5 such that $y v_{j}, y v_{j+2} \in E$ and $y v_{j+1} \notin E$. But then $\left\{y, v_{j}, v_{j+1}, v_{j+2}, x\right\}$ induces a paraglider, a contradiction.

Proof of (R9). Let $x \in T_{i}$.

(a): Suppose to the contrary that there exists a vertex $y \in T_{i+1} \cup T_{i-1} \cup Y \cup$ $\left(Z \backslash Z_{i+3}\right)$. First suppose that $y \in T_{i+1} \cup Y_{i} \cup Z_{i}$. Then since $y-v_{i}-x-v_{i+3}-v_{i+2}$ or $x-v_{i}-v_{i-1}-y-v_{i+2}$ is not a $P_{5}$, we have $x y \in E$. But then $\left\{v_{i}, v_{i+1}, y, v_{i-1}, x\right\}$ or $\left\{x, v_{i}, v_{i-2}, v_{i-1}, y\right\}$ induces a a paraglider. So $y \notin T_{i+1} \cup Y_{i} \cup Z_{i}$. Likewise, $y \notin T_{i-1} \cup Y_{i+1} \cup Z_{i+1}$. Next suppose that $y \in Y_{i+2}$. Then since $\left\{x, v_{i+1}, v_{i+2}, v_{i+3}, y\right\}$ does not induce a paraglider, we have $x y \in E$. But then $v_{i-1}-v_{i}-x-y-v_{i+2}$ is a $P_{5}$. So $y \notin Y_{i+2}$. Likewise, $y \notin Y_{i-1}$. Next suppose that $y \in Y_{i-2}$. Then since $\left\{x, v_{i}, v_{i-1}, v_{i-2}, y\right\}$ does not induce a paraglider, $x y \notin E$. But then $x-v_{i}-v_{i-1}-y-v_{i+2}$ is a $P_{5}$. So $y \notin Y_{i-2}$. Finally, suppose that $y \in Z_{i+2} \cup Z_{i-1}$. Up to symmetry, we may assume that $y \in Z_{i+2}$. Then since $\left\{x, v_{i}, v_{i-1}, v_{i-2}, y\right\}$ does not induce a paraglider, we have $x y \in E$. But then $\left\{x, v_{i+1}, v_{i+2}, v_{i+3}, y\right\}$ induces a paraglider, a contradiction. This proves item (a).

(b): Suppose that there is an edge $y z$ in one of the listed sets. If $y \in X_{i}$ and $z \in X_{i+2}$, then by (R/2), we have $x y, x z \notin E$; and then $z-y-v_{i-1}-v_{i}-x$ is a $P_{5}$. If $y \in X_{i+1}$ and $z \in X_{i-1}$, then by (R2 $\mathrm{c}$ ), we have $x y, x z \notin E$; and then $x$ $v_{i+1}-v_{i+2}-y-z$ is a $P_{5}$. If $y \in T_{i}$ and $z \in Z_{i+3}$, then $\left\{y, v_{i}, v_{i-1}, v_{i-2}, z\right\}$ induces a paraglider. These contradictions show that (b) holds.

Proof of (R[10). (a): Suppose not. Up to symmetry, we may assume that $q x \in$ $E$ and $q y \notin E$. Then either $\left\{q, x, y, v_{i+2}, v_{i}\right\}$ or $\left\{v_{i-1}, x, y, v_{i-2}, q\right\}$ induces a paraglider, a contradiction. So (a) holds.

(b): Otherwise, $x-v_{i}-q-v_{i+2}-y$ is a $P_{5}$. 
(c): Otherwise, $\left\{x, v_{i+1}, q, v_{i-1}, y\right\}$ induces a paraglider.

$(d)$ : This follows by item (c).

Proof of (R11). Suppose to contrary that there are adjacent vertices $x \in X_{i}$ and $y \in X_{i+2} \cup X_{i-2} \cup Y_{i+2} \cup Y_{i-2}$. We may assume, up to symmetry, that $y \in X_{i+2} \cup Y_{i+2}$. Now $\left\{v_{i}, v_{i+1}, y, v_{i-2}, v_{i-1}, x\right\}$ induces an $F_{1}$, a contradiction. So, $\left[X_{i}, X_{i+2} \cup X_{i-2} \cup Y_{i+2} \cup Y_{i-2}\right]=\emptyset$.

Now we show that $X=\emptyset$. Suppose to the contrary that $X \neq \emptyset$ and let $x \in X$, say $x \in X_{i}$ for some $i$. We claim that $x$ and $v_{i}$ are comparable. Since $G$ is $F_{1}$-free, $T=\emptyset$. Now by the preceding point, by the definition of $X_{i}$, and by (R3), (R/6), (R8 8 b), and since $G[C]$ is dominating, we see that $N_{G}(x)=\left\{v_{i+1}, v_{i-1}\right\} \cup X_{i+1} \cup X_{i-1} \cup Y_{i} \cup Y_{i+1} \cup Y_{i-1} \cup\left(Z \backslash Z_{i}\right) \cup A$, and $\overline{N_{G}(x)}=$ $\left\{v_{i+2}, v_{i-2}\right\} \cup\left(X_{i} \backslash\{x\}\right) \cup X_{i-2} \cup X_{i+2} \cup Y_{i+2} \cup Y_{i-2} \cup Z_{i}$. So, $N_{G}(x)=N_{G}\left(v_{i}\right)$ and $\overline{N_{G}(x)}=\overline{N_{G}\left(v_{i}\right)}$, and hence we conclude that $x$ and $v_{i}$ are comparable, a contradiction. So (R11) holds.

This completes the proof of Theorem 3

Theorem 4 Let $G$ be a ( $P_{5}$, paraglider)-free atom with no universal vertex. Suppose that $G$ contains $F_{1}$. Then $G$ has a stable set $S$ such that $G \backslash S$ is a bipartite graph or a bull. In particular, $G \backslash S$ is perfect.

Proof. Let $G$ be the given graph with vertex-set $V$ and edge-set $E$. First suppose that $G$ contains an $F_{1}^{\prime}$. Consider the graph $F_{1}^{\prime}$ as shown in Figure 1 and let $C=\left\{v_{1}, \ldots, v_{5}\right\}$. We use the same notation as in Theorem 3 and use the properties in Theorem 3 . Then by (R2 $\mathrm{a}$ ), $T_{1}=\left\{t_{1}\right\}$ and $T_{3}=\left\{t_{3}\right\}$. Moreover, by (R99), $T=\left\{t_{1}, t_{3}\right\}, Y=\emptyset$ and $Z=\emptyset$. Then since $Y=\emptyset$, any vertex in $A$ is a universal vertex of $G$ (by (R 80 ), and hence $A=\emptyset$. Also, by (R99 b), $\left[X_{1}, X_{3}\right]=\emptyset$ and $\left[X_{2}, X_{4}\right]=\emptyset$. Now, let us define $S:=\left\{t_{1}, t_{3}, v_{5}\right\} \cup X_{5}$, $S_{1}:=\left\{v_{1}, v_{3}\right\} \cup X_{1} \cup X_{3}$, and $S_{2}:=\left\{v_{2}, v_{4}\right\} \cup X_{2} \cup X_{4}$. Then by (R/3 a) and (R[2] c), the set $S:=\left\{t_{1}, t_{3}, v_{5}\right\} \cup X_{5}$ is a stable set. Also, by the preceding points and (R3 a), we see that $V(G) \backslash S=S_{1} \cup S_{2}$, and $S_{1}$ and $S_{2}$ are stable sets. Hence $G \backslash S$ is bipartite.

Suppose that $G$ contains no $F_{1}^{\prime}$. Consider the graph $F_{1}$ as shown in Figure 1 and let $C=\left\{v_{1}, \ldots, v_{5}\right\}$. We use the same notation as in Theorem 3 and use the properties in Theorem 3 Since $G$ has no $F_{1}^{\prime}$, by (R2 2 a), $T=\left\{t_{1}\right\}$. Then by (R9), the sets $Y, Z \backslash Z_{4},\left[X_{2}, X_{5}\right]$ and $\left[\left\{t_{1}\right\}, Z_{4}\right]$ are empty. Then since $Y=\emptyset$, any vertex in $A$ is a universal vertex of $G$ (by (R 8 (8) ), and hence $A=\emptyset$. Also, if there are adjacent vertices $x_{2} \in X_{2}$ and $x_{4} \in X_{4}$, then $\left\{v_{1}, v_{2}, v_{3}, x_{4}, v_{5}, t_{1}, x_{2}\right\}$ induces an $F_{2}$. So $\left[X_{2}, X_{4}\right]=\emptyset$. Likewise, $\left[X_{3}, X_{5}\right]=\emptyset$.

Suppose that $Z_{4}=\emptyset$. Then let us define $S:=\left\{t_{1}, v_{3}, v_{5}\right\} \cup X_{3} \cup X_{5}, S_{1}:=$ $\left\{v_{1}\right\} \cup X_{1}$, and $S_{2}:=\left\{v_{2}, v_{4}\right\} \cup X_{2} \cup X_{4}$. Then by (R/3 a) and (R/2 c), the set $S:=\left\{t_{1}, t_{3}, v_{5}\right\} \cup X_{5}$ is a stable set. Also, by the preceding points and (R/3 a), we see that $V(G) \backslash S=S_{1} \cup S_{2}$, and $S_{1}$ and $S_{2}$ are stable sets. Hence $G \backslash S$ is bipartite.

So let us assume that $Z_{4} \neq \emptyset$, and by (R[5a), $Z_{4}=\left\{z_{4}\right\}$. Then by (R96), $t_{1} z_{4} \notin E(G)$. Now we claim that $X_{j}=\emptyset$, for $j \neq 4$. Suppose not. Up to symmetry, we may assume that there exists a vertex $x \in X_{1} \cup X_{3}$. If $x \in X_{1}$, then by (R2 $2 \mathrm{c}$ ) and (R66), we have $t_{1} x \notin E$ and $x z_{4} \in E$. But then $v_{4}-t_{1}-v_{1}-z_{4^{-}}$ $x$ is a $P_{5}$. If $x \in X_{3}$, then since $\left\{v_{2}, v_{3}, v_{4}, x, z_{4}\right\}$ does not induce a paraglider, $x z_{4} \notin E$. But then $x-v_{4}-v_{3}-z_{4}-v_{1}$ is a $P_{5}$. So, we conclude that $X_{j}=\emptyset$, for 
$j \neq 4$. Now, by (R/3a) and (R6 b), the set $S:=\left\{z_{4}, v_{4}\right\} \cup X_{4}$ is a stable set such that $G \backslash S:=G\left[\left\{t_{1}, v_{5}, v_{1}, v_{2}, v_{3}\right\}\right]$ is a bull. This completes the proof of the theorem.

Theorem 5 Let $G$ be a $\left(P_{5}, F_{1}\right.$, paraglider $)$-free atom with no universal or pair of comparable vertices. Suppose that $G$ contains $F_{2}$. Then one of the following hold:

- $G$ is an induced subgraph of the complement of the Clebsch graph.

- $G$ has a good stable set.

- $G \in \mathcal{H}$.

Proof. Let $G$ be the given graph with vertex-set $V$ and edge-set $E$. Consider the graph $F_{2}$ as shown in Figure 1 and let $C=\left\{v_{1}, \ldots, v_{5}\right\}$. We use the same notation as in Theorem 3 and use the properties in Theorem [3. So $y_{2} \in Y_{2}$ and $y_{5} \in Y_{5}$, and $y_{2} y_{5} \in\left[Y_{2}, Y_{5}\right]$. Let $\left\{a_{1} b_{1}, a_{2} b_{2}, \ldots, a_{k} b_{k}\right\}$ denote the edges of $\left[Y_{2}, Y_{5}\right]$, and let $a_{1} b_{1}:=y_{2} y_{5}$. Moreover, let $Y_{2}^{*}:=Y_{2} \backslash\left\{a_{1}\right\}, Y_{2}^{\prime}:=Y_{2} \backslash$ $\left\{a_{1}, a_{2}, \ldots, a_{k}\right\}, Y_{5}^{*}:=Y_{5} \backslash\left\{b_{1}\right\}$ and $Y_{5}^{\prime}=Y_{5} \backslash\left\{b_{1}, b_{2}, \ldots, b_{k}\right\}$.

Since $G$ is $F_{1}$-free, $T=\emptyset$, and by (R11), $X=\emptyset$. Then we have the following:

Claim 5.1 For each $i \in\{1,2, \ldots, k\}$, the following hold:

(i) Any vertex in $Y_{2} \backslash\left\{a_{i}\right\}$ is adjacent to $a_{i}$ and non-adjacent to $b_{i}$ (and similarly, any vertex in $Y_{5} \backslash\left\{b_{i}\right\}$ is adjacent to $b_{i}$ and non-adjacent to $\left.a_{i}\right)$. In particular, $\left\{a_{1}, \ldots, a_{k}\right\}$ and $\left\{b_{1}, \ldots, b_{k}\right\}$ are cliques.

(ii) $\left[Y_{3},\left\{b_{i}\right\}\right]$ is complete and $\left[Y_{4},\left\{a_{i}\right\}\right]$ is complete.

(iii) $\left[Y_{3}, Y_{5} \backslash\left\{b_{i}\right\}\right]=\emptyset$ and $\left[Y_{4}, Y_{2} \backslash\left\{a_{i}\right\}\right]=\emptyset$.

(iv) Any vertex $a \in A$ is either complete to $\left\{a_{i}, b_{i}\right\}$ or anti-complete to $\left\{a_{i}, b_{i}\right\}$. Moreover, if $a \in A$ and if there exists an index $i$ such that $a$ is anticomplete to $\left\{a_{i}, b_{i}\right\}$, then a is complete to $\left(Y_{2} \backslash\left\{a_{i}\right\}\right) \cup\left(Y_{5} \backslash\left\{b_{i}\right\}\right)$.

Proof. (i): Let $x \in Y_{2} \backslash\left\{a_{i}\right\}$ be arbitrary. If $x a_{i} \notin E$, then since $\left\{x, v_{1}, a_{i}, v_{3}, b_{i}\right\}$ does not induce a paraglider, we have $x b_{i} \notin E$, and then $x-v_{2}-a_{i}-b_{i}-v_{5}$ is a $P_{5}$. So, $x a_{i} \in E$. Moreover, since $\left\{x, v_{3}, v_{4}, b_{i}, a_{i}\right\}$ does not induces a paraglider, we have $x b_{i} \notin E$. Thus any vertex in $Y_{2} \backslash\left\{a_{i}\right\}$ is adjacent to $a_{i}$ and non-adjacent to $b_{i}$. Likewise, any vertex in $Y_{5} \backslash\left\{b_{i}\right\}$ is adjacent to $b_{i}$ and non-adjacent to $a_{i}$. So (i) holds.

(ii): If there is a vertex $x \in Y_{3}$ such that $x b_{i} \notin E$, then by (R44 b), $x a_{i} \in E$, and then $\left\{a_{i}, v_{3}, v_{4}, b_{i}, x\right\}$ induces a paraglider which is a contradiction. So $\left[Y_{3},\left\{b_{i}\right\}\right]$ is complete. Likewise, $\left[Y_{4},\left\{a_{i}\right\}\right]$ is complete. Thus (ii) holds.

(iii): Suppose to the contrary that there are adjacent vertices $x \in Y_{3}$ and $y \in Y_{5} \backslash\left\{b_{i}\right\}$. By item $(i)$, we have $y b_{i} \in E$, and by item $(i i), x b_{i} \in E$. Now, $\left\{x, y, v_{1}, v_{2}, b_{i}\right\}$ induces a paraglider which is a contradiction. So (iii) holds.

$(i v)$ : This follows by item $(i),(\mathrm{R}[10 \mathrm{a})$ and $(\mathrm{R} 10 \mathrm{~b})$.

Next we have the following:

Claim 5.2 $Y_{1}$ is a clique, $\left|Y_{3}\right| \leq 1,\left|Y_{4}\right| \leq 1$, and $\left[Y_{1}, Y_{3} \cup Y_{4}\right]$ is complete. 
Proof. First, since $y_{2} \in Y_{2}$ and $y_{5} \in Y_{5}$, by (R4 4 d), $Y_{1}$ is a clique. Next, we know by Claim [5.1(ii) that $\left[Y_{3},\left\{b_{1}\right\}\right]$ and $\left[Y_{4},\left\{a_{1}\right\}\right]$ are complete. So by (R4] e), we have $\left|Y_{3}\right| \leq 1$ and $\left|Y_{4}\right| \leq 1$. Finally, suppose to the contrary that there are non-adjacent vertices $y_{1} \in Y_{1}$ and $y_{3} \in Y_{3}$. We know by (R4 b) that $y_{1} b_{1} \in E$, and by Claim [5.1(ii) that $y_{3} b_{1} \in E$. Now $\left\{b_{1}, v_{1}, v_{2}, y_{3}, y_{1}\right\}$ induces a paraglider, a contradiction. So $\left[Y_{1}, Y_{3}\right]$ is complete. Likewise, $\left[Y_{1}, Y_{4}\right]$ is complete.

For each $i \in\{1,2, \ldots, k\}$, let $A_{i}^{\prime}:=\left\{x \in A \mid x\right.$ is anti-complete to $\left\{a_{i}, b_{i}\right\}$ and is complete to $\left(Y_{2} \backslash\left\{a_{i}\right\} \cup\left(Y_{5} \backslash\left\{b_{i}\right\}\right)\right\}$. Let $A^{\prime}:=A_{1}^{\prime} \cup \cdots \cup A_{k}^{\prime}$. Let $A^{\prime \prime}:=$ $\left\{x \in A \mid x\right.$ is complete to $\left.\left\{a_{1}, \ldots, a_{k}, b_{1}, \ldots, b_{k}\right\}\right\}$. Then by Claim 5.1(iv), $A=A^{\prime} \cup A^{\prime \prime}$. Moreover, we have the following claim.

Claim 5.3 The following hold: (i) For each $i \in\{1,2, \ldots, k\},\left|A_{i}^{\prime}\right| \leq 1$. (ii) $\left[A^{\prime}\right.$, $\left.Y_{1} \cup Y_{3} \cup Y_{4}\right]=\emptyset$. (iii) $\left[A^{\prime \prime}, Y_{1} \cup Y_{3} \cup Y_{4}\right]$ is complete.

Proof. (i): Suppose to the contrary that $\left|A_{i}^{\prime}\right| \geq 2$ and let $x, x^{\prime} \in A_{i}^{\prime}$. Since $A_{i}^{\prime} \subseteq A$ and $A$ is a clique (by (R8 $\mathrm{a}$ ), $x x^{\prime} \in E$. But, then $\left\{x, v_{1}, a_{i}, v_{3}, x^{\prime}\right\}$ induces a paraglider. So (i) holds.

(ii): Suppose not. Then there are adjacent vertices $q \in A^{\prime}$ and $y \in Y_{1} \cup Y_{3} \cup Y_{4}$. Since $q \in A^{\prime}$, there exists a pair $\left\{a_{i}, b_{i}\right\}$ such that $q$ is anti-complete to $\left\{a_{i}, b_{i}\right\}$. Now if $y \in Y_{1}$, then by (R母 $\left.\mathrm{b}\right), y b_{i} \in E$, and then $\left\{v_{1}, q, v_{4}, b_{i}, y\right\}$ induces a paraglider which is a contradiction. So $y \in Y_{3} \cup Y_{4}$. Then since $\left[Y_{3},\left\{b_{i}\right\}\right]$ and $\left[Y_{4},\left\{a_{i}\right\}\right]$ are complete (by Claim [5.1(ii)), we have a contradiction to (R10 a). So (ii) holds.

(iii): Suppose not. Then there are non-adjacent vertices $q \in A^{\prime \prime}$ and $y \in$ $Y_{1} \cup Y_{3} \cup Y_{4}$. Since $q \in A^{\prime \prime}, q$ is complete to $\left\{a_{1}, b_{1}\right\}$. Now if $y \in Y_{1}$, then by (R4 $\mathrm{b}$ ), $y b_{1} \in E$, and then $\left\{v_{5}, y, v_{2}, q, b_{1}\right\}$ induces a paraglider which is a contradiction. So $y \in Y_{3} \cup Y_{4}$. Then since $\left[Y_{3},\left\{b_{1}\right\}\right]$ and $\left[Y_{4},\left\{a_{1}\right\}\right]$ are complete (by Claim [5.1(ii)), we have a contradiction to (R10 a). So (iii) holds.

Claim 5.4 Let $x \in A^{\prime \prime}$. Then either $x$ is complete to $Y_{2}$ or $x$ is complete to $Y_{5}$.

Proof. Suppose not. Then there exist vertices $p \in Y_{2}^{\prime}$ and $q \in Y_{5}^{\prime}$ such that $x p, x q \notin E$. But, then $p-v_{2}-x-v_{5}-q$ is a $P_{5}$.

Suppose that $A^{\prime \prime} \neq \emptyset$, and let $x \in A^{\prime \prime}$. By Claim 5.4 and up to symmetry, we may assume that $x$ is complete to $Y_{5}$. Then using (R 8 and Claim 5.3 and since $x$ is not universal, we conclude that $x$ has a non-neighbor in $Y_{2}$. Moreover, by (R $10 \mathrm{~d}), \overline{N(x)} \cap Y_{2}$ is a stable set. Now let us define $S:=\{x\} \cup\left(\overline{N(x)} \cap Y_{2}\right)$. Then since $\left[\{x\}, V(G) \backslash\left(\overline{N(x)} \cap Y_{2}\right)\right]$ is complete, by (R4a), we see that $S$ is a good stable set of $G$. So, we may assume that $A^{\prime \prime}=\emptyset$.

Claim 5.5 The following hold: (i) If $Y_{2}^{*} \cup Y_{5}^{*} \neq \emptyset$, then $Z_{1}=\emptyset$. (ii) If $Y_{2}^{*} \neq \emptyset$, then $Y_{3}=\emptyset$. (iii) If $Y_{5}^{*} \neq \emptyset$, then $Y_{4}=\emptyset$.

Proof. To prove the claim, we show that if $Y_{2}^{*} \neq \emptyset$, then $Z_{1} \cup Y_{3}=\emptyset$, and the other cases follow by symmetry. Let $x \in Y_{2}^{*}$. Suppose to the contrary that $Z_{1} \cup Y_{3} \neq \emptyset$, and let $y \in Z_{1} \cup Y_{3}$. We know by Claim [5.1(i) that $a_{1} x \in E$ and $b_{1} x \notin E$. Now if $y \in Z_{1}$, then by (R/7 b), we have $y a_{1}, y b_{1}, y x \notin E$. But then $y-v_{5}-b_{1}-a_{1}-x$ is a $P_{5}$. So $y \in Y_{3}$. Then by (R近b), $x y \in E$, and by Claim [5.1(ii), 
$y b_{1} \in E$. But then $\left\{x, y, b_{1}, v_{1}, v_{2}\right\}$ induces a paraglider which is a contradiction. So, $Z_{1} \cup Y_{3}=\emptyset$.

Claim 5.6 If $Z_{1} \cup Z_{2} \cup Z_{5} \cup A_{1}^{\prime} \neq \emptyset$, then $\left|Y_{1}\right| \leq 1$.

Proof. Suppose not. Let $x, y \in Y_{1}$ and $z \in Z_{1} \cup Z_{2} \cup Z_{5} \cup A_{1}^{\prime}$. We know by Claim 5.2 that $Y_{1}$ is a clique and so $x y \in E$. Moreover, by (R4 4 b), $\{x, y\}$ is complete to $\left\{a_{1}, b_{1}\right\}$. If $z \in Z_{1}$, then $z x, x y \in E$ (by (R/7 7 )), and then by (R77b), $\left\{x, z, v_{3}, a_{1}, y\right\}$ induces a paraglider. If $z \in Z_{2} \cup Z_{5}$, then $z x, x y \notin E$ (by (R/7 b)). Also if $z \in Z_{2}$, then $z a_{1} \in E$ (by (R/7a)), and if $z \in Z_{5}$, then $z b_{1} \in E$ (by (R/7 a)). But then either $\left\{x, v_{5}, z, a_{1}, y\right\}$ or $\left\{x, v_{2}, z, b_{1}, y\right\}$ induces a paraglider. If $z \in A_{1}^{\prime}$, then by Claim[5.3(ii), $z x, z y \notin E$. But then $\left\{x, v_{2}, z, v_{5}, y\right\}$ induces a paraglider.

Suppose that $Y_{2}^{*} \cup Y_{5}^{*}=\emptyset$. Since $A^{\prime \prime}=\emptyset, A=A_{1}^{\prime}$. If $Y_{3} \cup Y_{4} \cup Z_{1} \cup Z_{2} \cup$ $Z_{5} \cup A_{1}^{\prime} \neq \emptyset$ or if $\left|Y_{1}\right| \leq 1$, then by Claims 5.2 and [5.6] and (R4 4 e) we conclude that $Y$ is a clique with $|Y| \leq 5$. So $|V(G)|=\left|V\left(C_{5}\right)\right|+|Z|+|Y|+\left|A_{1}^{\prime}\right| \leq 16$, and we see that in this case, $G$ is an induced subgraph of the complement of the Clebsch graph. If $Y_{3} \cup Y_{4} \cup Z_{1} \cup Z_{2} \cup Z_{5} \cup A_{1}^{\prime}=\emptyset$ and $\left|Y_{1}\right| \geq 2$, then since $Y_{1}$ is complete to $\left\{a_{1}, b_{1}\right\}$ (by ( $\left.\mathrm{R} 4 \mathrm{~b}\right)$ ), we see that $\omega(G) \geq 5$, and hence $\left\{v_{1}\right\}$ is a good stable set of $G$.

So suppose that $Y_{2}^{*} \cup Y_{5}^{*} \neq \emptyset$. We may assume, up to symmetry, that $Y_{2}^{*} \neq \emptyset$. Let $p \in Y_{2}^{*}$. Then by Claim [5.5, $Z_{1} \cup Y_{3}=\emptyset$. Further we have the following.

Claim 5.7 We have: Either $Y_{1}=\emptyset$ or $Y_{4} \cup Z_{2} \cup Z_{5} \cup A^{\prime}=\emptyset$.

Proof. Suppose not. Let $x \in Y_{1}$ and $y \in Y_{4} \cup Z_{2} \cup Z_{5} \cup A^{\prime}$. Then by (R44 $\mathrm{b}$ ), $x b_{1}, p x \in E$. Now: If $y \in Y_{4}$, then $y x \in E$ ( by Claim [5.2), $p y \notin E$ (by Claim [5.1(iii)). But then $\left\{x, y, v_{3}, p, v_{2}\right\}$ induces a paraglider. If $y \in Z_{2}$, then by (R77), $y b_{1}, y p \in E$ and $y x \notin E$. But then $\left\{p, x, v_{5}, y, b_{1}\right\}$ induces a paraglider. If $y \in Z_{5}$, by (R/7), $y b_{1}, y p \in E$ and $y x \notin E$. But, then $\left\{p, x, b_{1}, y, v_{2}\right\}$ induces a paraglider. So, we may assume that $y \in A^{\prime}$. Then there exists a pair $\left\{a_{i}, b_{i}\right\}$ such that $y$ is anti-complete to $\left\{a_{i}, b_{i}\right\}$. By Claim [5.1(i), $p a_{i} \in E$ and $p b_{i} \notin E$. Then since $p-v_{2}-y-v_{5}-b_{i}$ is not a $P_{5}$, we have $p y \in E$. Also, by Claim 5.3 $x y \notin E$. But now $\left\{p, x, v_{5}, y, v_{2}\right\}$ induces a paraglider which is a contradiction. So the claim holds.

First suppose that $Y_{1} \neq \emptyset$. Then by Claim 5.7, $Y_{4} \cup Z_{2} \cup Z_{5} \cup A^{\prime}=\emptyset$. But then $\left\{v_{1}\right\}$ is a good stable set of $G$. So, we may assume that $Y_{1}=\emptyset$.

Next suppose that $Y_{4} \neq \emptyset$. Then by Claim [5.2] we let $Y_{4}=\left\{y_{4}\right\}$. Also, by Claim $5.5 .5 Y_{5}^{*}=\emptyset$. Moreover, we show that $Z_{3}=\emptyset$. Suppose not, and let $z_{3} \in Z_{3}$. Then by (R/7b), $z_{3} p, z_{3} y_{4} \notin E$. But then $y_{4}-v_{4}-z_{3}-v_{1}-p$ is a $P_{5}$. So, $Z_{3}=\emptyset$. Now, in this case, we see that there is a good stable set of $G$ as follows: If $Z=\emptyset$, then $\left\{y_{4}, v_{2}\right\}$ is a good stable set. So $Z \neq \emptyset$. If $Z_{2} \neq \emptyset$, then by (R[5), (R77a), and (R/8), $\left[Z_{2}, V(G) \backslash\left\{v_{2}\right\}\right]$ is complete, and hence $\left\{z_{2}, v_{2}\right\}$ is a good stable set of $G$. So $Z_{2}=\emptyset$. Next if $Z_{4} \neq \emptyset$, then since $\left[Z_{4}, V(G) \backslash\left\{v_{4}, b_{1}\right\}\right]$ is complete, $\left\{z_{4}, v_{4}\right\}$ is a good stable set of $G$. So $Z_{4}=\emptyset$. Finally, if $Z_{5} \neq \emptyset$, then since $\left[Z_{4}, V(G) \backslash\left\{b_{1}\right\} \cup Y_{4}\right]$ is complete, $\left\{z_{5}, v_{5}\right\}$ is a good stable set of $G$.

So, we may assume that $Y_{4}=\emptyset$. If $Z_{2} \neq \emptyset$, then since $\left[Z_{2}, V(G) \backslash\left(\left\{v_{2}\right\} \cup Z_{3}\right)\right]$ is complete, we see that $\left\{z_{2}, v_{2}\right\}$ is a good stable set of $G$. So $Z_{2}=\emptyset$. Likewise, $Z_{5}=\emptyset$. Then we define $Q_{1} \cup R_{1}:=\left\{a_{1}, a_{2}, \ldots, a_{k}, v_{2}, v_{3}\right\} \cup Y_{2}^{\prime} \cup Z_{4}, Q_{2} \cup R_{2}:=$ 
$\left\{b_{1}, b_{2}, \ldots, b_{k}, v_{4}, v_{5}\right\} \cup Y_{5}^{\prime} \cup Z_{3}$, and $S:=\left\{v_{1}\right\} \cup A^{\prime}$. Now, it is easy see that $G \in \mathcal{H}$.

This completes the proof of the theorem.

Theorem 6 Let $G$ be a $\left(P_{5}, F_{1}, F_{2}\right.$,paraglider)-free atom with no universal or pair of comparable vertices. Suppose that $G$ contains $F_{3}$. Then $G$ has a stable set $S$ such that either $S$ is good or $G \backslash S$ is perfect.

Proof. Let $G$ be the given graph with vertex-set $V$ and edge-set $E$. Consider the graph $F_{3}$ as shown in Figure 1 and let $C=\left\{v_{1}, \ldots, v_{5}\right\}$. We use the same notation as in Theorem 3 and use the properties in Theorem 3 . So $y_{1} \in Y_{1}$ and $z_{1} \in Z_{1}$. Since $G$ is $F_{1}$-free, $T \cup X=\emptyset$ (by $(\mathrm{R}[11)$ ). Moreover, we have the following:

Claim 6.1 For each $i$, we have $\left[Y_{i}, Y_{i+2}\right]=\emptyset$.

Proof. Suppose to the contrary that there are adjacent vertices, say $x \in Y_{i}$ and $y \in Y_{i+2}$. Then $\left\{v_{1}, v_{2}, v_{3}, v_{4}, v_{5}, x, y\right\}$ induces an $F_{2}$ which is a contradiction. So the claim holds.

Claim 6.2 For each $i$, we have either $Z_{i}=\emptyset$ or $Y_{i-1} \cup Y_{i+1}=\emptyset$.

Proof. Suppose not. Up to symmetry, let $z \in Z_{i}$ and $y \in Y_{i+1}$. Then by (R/7), $z y \notin E$. But, then $\left\{v_{1}, v_{2}, v_{3}, v_{4}, v_{5}, z, y\right\}$ induces an $F_{2}$.

Since $z_{1} \in Z_{1}$ and $y_{1} \in Y_{1}$, by Claim 6.2 the sets $Y_{2}, Y_{5}, Z_{2}$ and $Z_{5}$ are empty. If $A=\emptyset$, then up to symmetry, we have three cases: (a) $Z_{3} \neq \emptyset$ and $Z_{4} \neq \emptyset$. (b) $Z_{3} \neq \emptyset$ and $Z_{4}=\emptyset$. (c) $Z_{3}=\emptyset$ and $Z_{4}=\emptyset$. In Case (a), $Y_{3}=Y_{4}=\emptyset$, and hence $G-\left\{v_{2}, v_{4}\right\}$ is a $\overline{P_{3}}$-free expansion of a perfect graph, and hence perfect. In Case (b) and in Case (c), $G-\left\{v_{2}, v_{5}\right\}$ is a $\overline{P_{3}}$-free expansion of a $P_{4}$, and hence perfect.

So suppose that $A \neq \emptyset$. First let us assume that $\left[A, Y_{1}\right]$ is not complete. Then there exists a vertex $x \in A$ that has a non-neighbor in $Y_{1}$, say $y \in Y_{1}$. If $x$ has a non-neighbor $y^{\prime} \in Y_{3} \cup Y_{4}$, then $y-v_{1}-x-v_{3}-y^{\prime}$ or $y-v_{1}-x-v_{4}-y^{\prime}$ is a $P_{5}$. So, by $\left(\mathrm{R}[8),\left[\{x\}, V(G) \backslash Y_{1}\right]\right.$ is complete. Then by (R[10] d), $\{x\} \cup\left(\overline{N(x)} \cap Y_{1}\right)$ is a good stable set of $G$. So we may assume that $\left[A, Y_{1}\right]$ is complete. Then since $G$ has no universal vertex, by (R/8), $x$ has a non-neighbor in $Y_{3} \cup Y_{4}$. Then $\{x\} \cup\left(\overline{N(x)} \cap Y_{3}\right)$ or $\{x\} \cup\left(\overline{N(x)} \cap Y_{4}\right)$ is a good stable set of $G$. This complete the proof of the theorem.

Theorem 7 Let $G$ be a $\left(P_{5}, F_{1}, F_{2}, F_{3}\right.$,paraglider $)$-free atom with no universal or pair of comparable vertices. Suppose that $G$ contains a $C_{5}$. Then one of the following hold:

- $G$ is an induced subgraph of the complement of the Petersen graph.

- $G$ is an $\overline{P_{3}}$-free expansion of $C_{5}$.

- $G$ has a stable set $S$ such that $G \backslash S$ is perfect. 
Proof. Let $G$ be the given graph with vertex-set $V$ and edge-set $E$. Suppose that $G$ contains $C_{5}$ with vertex set $C=\left\{v_{1}, \ldots, v_{5}\right\}$. We use the same notation as in Theorem 3 and use the properties in Theorem 3 . Since $G$ is $F_{1}$-free, $T \cup X=\emptyset$ (by $\left(\mathrm{R}[11)\right.$ ). Since $G$ is $F_{2}$-free, we have, for each $i,\left[Y_{i}, Y_{i+2}\right]=\emptyset$. Since $G$ is $F_{3}$-free, $[A, Y]$ is complete. So by (R[8), any vertex in $A$ is a universal vertex of $G$ and hence $A=\emptyset$. Moreover, if $z_{i} \in Z$, then since $G$ is $\left(F_{2}, F_{3}\right)$-free, by (R/7), we have $Y_{j}=\emptyset$, for $j \in\{i-1, i, i+1\}$.

If $Z=\emptyset$, then $G$ is a $\overline{P_{3}}$-free expansion of $C_{5}$ (by (R 4 ). So let us assume that $Z \neq \emptyset$. If there exists an $i$ such that $z_{i}, z_{i+2} \in Z$, then $Y=\emptyset$. Now by (R[5), $G$ is an induced subgraph of the complement of the Petersen graph. Finally up to symmetry, let us assume that $Z=\left\{z_{1}\right\}$ or $Z=\left\{z_{1}, z_{2}\right\}$. (a) If $Z=\left\{z_{1}\right\}$, then $Y_{j}=\emptyset$, for $j \in\{1,2,5\}$, and by (R[7]), $\left[Y_{3} \cup Y_{4}, Z\right]$ is complete. So, by (R 4 a), $G \backslash\left\{v_{1}\right\}$ is a $\overline{P_{3}}$-free expansion of a perfect graph, and hence perfect. (b) If $Z=\left\{z_{1}, z_{2}\right\}$, then $Y_{j}=\emptyset$, for $j \in\{1,2,3,5\}$, and by (R/7), $\left[Y_{4}, Z\right]$ is complete. Then we see that by (R4a), $G \backslash\left\{v_{1}, v_{3}\right\}$ is a $\overline{P_{3}}$-free expansion of a perfect graph, and hence perfect. This completes the proof of the theorem.

\section{Coloring $\left(P_{5}\right.$, paraglider $)$-free graphs}

Given a graph $G$ and a proper homogeneous set $X$ in $G$, let $G / X$ be the graph obtained by replacing $X$ with a clique $Q$ of size $\omega(X)$ (i.e., $G / X$ is obtained from $G \backslash X$ and $Q$ by adding all edges between $Q$ and the vertices of $V(G) \backslash X$ that are adjacent to $X$ in $G$ ). The proof of the following lemma is very similar to that of Lemma 3.1 of [10] and we omit the details.

Lemma 1 ([10]) In a graph $G$ let $X$ be a proper homogeneous set such that $G[X]$ is perfect. Then $\omega(G)=\omega(G / X)$ and $\chi(G)=\chi(G / X)$.

Let $\mathcal{C}_{5}$ be the class of graphs that are $\overline{P_{3}}$-free expansions of $C_{5}$, and let $\mathcal{C}_{5}^{*}$ be the class of graphs that are clique expansions of $C_{5}$.

Let $\mathcal{H}^{*}$ be the class of graphs $G \in \mathcal{H}$ such that, with the notation as in Section 1 , the two sets $R_{1}$ and $R_{2}$ are cliques.

Since $\overline{P_{3}}$-free graphs are perfect, the following lemma (Lemma 21) can be proved using Lemma 1, and the proof is very similar to that of Lemma 3.3 of [10, so we omit the details.

Lemma 2 (10]) For every graph $G$ in $\mathcal{C}_{5}$ (resp. $G$ in $\left.\mathcal{H}\right)$ there is a graph $G^{*}$ in $\mathcal{C}_{5}^{*}$ (resp. $G^{*}$ in $\mathcal{H}^{*}$ ) such that $\omega(G)=\omega\left(G^{*}\right)$ and $\chi(G)=\chi\left(G^{*}\right)$.

Lemma 3 ([10]) Let $G$ be a clique expansion of $C_{5}$. Then $\chi(G) \leq\left\lceil\frac{5 \omega(G)}{4}\right\rceil$.

For any fixed integer $k \geq 2$, let $G_{k}$ be the graph defined as follows.

- $V\left(G_{k}\right)$ can be partitioned into three cliques $Q_{1}:=\left\{a_{1}, a_{2} \ldots, a_{k}\right\}, Q_{2}:=$ $\left\{b_{1}, b_{2}, \ldots, b_{k}\right\}$, and $S:=\left\{s_{1}, s_{2}, \ldots, s_{k}\right\}$ such that $\left[Q_{1}, Q_{2}\right]$ is a perfect matching, say $\left\{a_{1} b_{1}, a_{2} b_{2}, \ldots, a_{k} b_{k}\right\}$.

- There exists an injective function $f: S \rightarrow\{1,2, \ldots, k\}$ such that for each vertex $x \in S,\{x\}$ is anti-complete to $\left\{a_{f(x)}, b_{f(x)}\right\}$, and is complete to $\left(Q_{1} \cup Q_{2}\right) \backslash\left\{a_{f(x)}, b_{f(x)}\right\}$.

- No other edges in $G$. 
Lemma 4 For each integer $k \geq 2, \chi\left(G_{k}\right) \leq\left\lceil\frac{3 k}{2}\right\rceil$.

Proof. Without loss of generality, we may assume that for each $i \in\{1,2 \ldots, k\}$, $s_{i}$ is anti-complete to $\left\{a_{i}, b_{i}\right\}$ and complete to $\left(Q_{1} \cup Q_{2}\right) \backslash\left\{a_{i}, b_{i}\right\}$. We consider two cases depending on whether $k$ is even or not.

Suppose first that $k=2 t$ for some $t \geq 1$. Now we color $G_{2 t}$ using $3 t$ colors as follows:

- Color $s_{1}, s_{2}, \ldots, s_{t}$ with colors $1,2, \ldots, t$, respectively.

- Color $s_{t+1}, s_{t+2}, \ldots, s_{2 t}$ with colors $t+1, t+2, \ldots, 2 t$, respectively.

- Color $a_{1}, a_{2}, \ldots, a_{t}$ with colors $2 t+1,2 t+2, \ldots, 3 t$, respectively.

- Color $a_{t+1}, a_{t+2}, \ldots, a_{2 t}$ with colors $t+1, t+2, \ldots, 2 t$, respectively.

- Color $b_{1}, b_{2}, \ldots, b_{t}$ with colors $1,2, \ldots, t$, respectively.

- Color $b_{t+1}, b_{t+2}, \ldots, b_{2 t}$ with colors $2 t+1,2 t+2, \ldots, 3 t$, respectively.

Then it can be easily checked that the above is a $3 t$-coloring of $G_{2 t}$.

Next suppose that $k=2 t+1$, for some $t \geq 1$. Then $\left\lceil\frac{3 k}{2}\right\rceil=3 t+2$. Observe that $G_{2 t+1} \backslash\left\{s_{2 t+1}, a_{2 t+1}, b_{2 t+1}\right\}$ is isomorphic to $G_{2 t}$. Therefore, $\chi\left(G_{2 t+1}\right) \leq$ $3 t+2$.

Theorem 8 If $G \in \mathcal{H}^{*}, \chi(G) \leq \frac{3 \omega(G)}{2}$.

Proof. Let $G$ be partitioned into $Q_{1}, Q_{2}, R_{1}, R_{2}$ and $S$. Without loss of generality, we may assume that for each $1 \leq i \leq|S|, s_{i}$ is complete to $\left(Q_{1} \cup\right.$ $\left.Q_{2}\right) \backslash\left\{a_{i}, b_{i}\right\}$ and is anti-complete to $\left\{a_{i}, b_{i}\right\}$. Let $r=\max \left\{\left|R_{1}\right|,\left|R_{2}\right|\right\}$. Since $R_{i} \cup Q_{i}$ is a clique for $i \in\{1,2\}, \omega(G) \geq k+r$. Obviously,

$$
\chi(G) \leq \chi\left(G\left[Q_{1} \cup Q_{2} \cup S\right]\right)+\chi\left(G\left[R_{1} \cup R_{2}\right]\right) .
$$

Since $|S| \leq k, G\left[Q_{1} \cup Q_{2} \cup S\right]$ is an induced subgraph of $G_{k}$. By Lemma 4, $\chi\left(G\left[Q_{1} \cup Q_{2} \cup S\right]\right) \leq\left\lceil\frac{3 k}{2}\right\rceil$. On the other hand, since $R_{1}$ and $R_{2}$ are clique, $\chi\left(G\left[R_{1} \cup R_{2}\right]\right) \leq r$. If $r \geq 1$, then $r+\frac{1}{2} \leq \frac{3 r}{2}$. Therefore, $\chi(G) \leq\left(\frac{3 k}{2}+\frac{1}{2}\right)+r \leq$ $\frac{3 k}{2}+\frac{3 r}{2} \leq \frac{3 \omega(G)}{2}$. So we may assume that $r=0$. Observe that $\omega(G) \in\{k, k+1\}$ and that if $\omega(G)=k$, then $|S| \leq k-2$. If $S=\emptyset$, then $\chi(G) \leq k \leq \omega(G)$. So, let $S=\left\{s_{1}, s_{2}, \ldots, s_{t}\right\}$ for some $1 \leq t \leq k$. Let

$$
\begin{aligned}
G^{\prime} & =G\left[\left\{a_{1}, a_{2}, \ldots a_{t}\right\} \cup\left\{b_{1}, b_{2}, \ldots b_{t}\right\} \cup\left\{s_{1}, s_{2}, \ldots, s_{t}\right\}\right], \text { and } \\
G^{\prime \prime} & =G\left[\left\{a_{t+1}, \ldots, a_{k}\right\} \cup\left\{b_{t+1}, \ldots, a_{k}\right\}\right] .
\end{aligned}
$$

Observe that $\chi\left(G^{\prime \prime}\right)=k-t$. Since $G^{\prime}$ is isomorphic to $G_{t}$, it follows from Lemma 4 that $\chi\left(G^{\prime}\right) \leq\left\lceil\frac{3 t}{2}\right\rceil \leq \frac{3 t}{2}+\frac{1}{2}$. Therefore, $\chi(G) \leq \chi\left(G^{\prime}\right)+\chi\left(G^{\prime \prime}\right) \leq$ $\left(\frac{3 t}{2}+\frac{1}{2}\right)+(k-t)=k+\frac{t}{2}+\frac{1}{2}$. Now if $\omega(G)=k$, then since $t \leq k-2$, we have $\chi(G) \leq k+\frac{k-2}{2}+\frac{1}{2}=\frac{3 k}{2}-\frac{1}{2}<\frac{3}{2} \omega(G)$, and if $\omega(G)=k+1$, then $\chi(G) \leq k+\frac{k}{2}+\frac{1}{2}<\frac{3}{2} \omega(G)$.

Let $G$ be a graph and $v \in V(G)$. We say that $G^{\prime}$ is obtained from $G$ by adding a smaller vertex $u$ if $N(u)$ is a non-empty subset of $N(v)$ in $G^{\prime}$. Let $\mathcal{B}$ be the set of graphs that consists of the complement of the Clebsch graph and the graph obtained from the complement of the Clebsch graph by deleting a vertex. We note that any graph $G \in \mathcal{B}$ has $\chi(G)=\left\lceil\frac{3 \omega(G)}{2}\right\rceil$ and the ceiling is necessary. Moreover, it is not hard to verify that these are the only induced subgraphs of the complement of the Clebsch graph that satisfy this property (This fact 
and Lemma 5 below are verified by a computer program due to Owen Merkel). Let $\mathcal{G}$ be the class of ( $P_{5}$, paraglider)-free graphs that can be obtained from a graph in $\mathcal{B}$ by a sequence of adding a smaller vertex. We say that a graph $G$ is awesome if for every non-empty clique $K$ of $G$, there exists an induced $P_{4}:=$ $v-x-y-z$ such that $v \in K$ and $x, y, z \notin K$. Then we have the following lemma and its proof is verified easily by a computer program.

Lemma 5 Every graph $G \in \mathcal{B}$ is awesome.

Lemma 6 Every graph $G \in \mathcal{G}$ is awesome.

Proof. Let $G \in \mathcal{G}$. Then $G$ is obtained from a graph $B \in \mathcal{B}$ by adding smaller vertices $u_{1}, \ldots, u_{k}$ sequentially. We prove the lemma by induction on $k$. If $k=0$, then the lemma holds by Lemma 5. Suppose now that the lemma holds for all graphs in $\mathcal{G}$ that are obtained from a graph $B \in \mathcal{B}$ by adding $k-1$ vertices for some $k \geq 1$. Let $G^{\prime}=G-u_{k}$. By the inductive hypothesis, $G^{\prime}$ is awesome, i.e., for every non-empty clique $K$ of $G^{\prime}$ there exists an induced $P_{4}:=v-x-y-z$ such that $v \in K$ and $x, y, z \notin K$.

Now let $K$ be a clique of $G$. If $K \backslash\left\{u_{k}\right\}$ is non-empty, then it follows from the inductive hypothesis that a desired $P_{4}$ exists for $K$. It remains to consider the case that $K=\left\{u_{k}\right\}$, i.e., to show that in $G$ there is an induced $P_{4}:=u_{k}-x-y-z$. Let $v \in V\left(G^{\prime}\right)$ be the vertex such that $N_{G}\left(u_{k}\right) \subseteq N_{G}(v)$.

Suppose first that $N_{G}\left(u_{k}\right)=N_{G}(v)$. Since $G^{\prime}$ is awesome, there exists an induced path $v-x-y-z$ in $G^{\prime}$ (consider the clique $\{v\}$ ). Thus, $u_{k}-x-y-z$ is a desired $P_{4}$.

So, we may assume that there exists a vertex $d \in V\left(G^{\prime}\right)$ such that $d$ is adjacent to $v$ but not to $u_{k}$. Suppose that $u_{k}$ has two non-adjacent neighbors $s$ and $t$ in $G$. Since $\left\{v, s, u_{k}, t, d\right\}$ does not induce a paraglider, $d$ is not adjacent

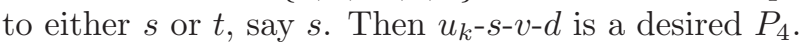

So, $N_{G}\left(u_{k}\right)$ is a clique. Let $w \in V\left(G^{\prime}\right)$ be a neighbor of $u$. Since $G^{\prime}$ is awesome, there exists an induced $P_{4}:=w-x-y-z$ in $G^{\prime}$. Since $N_{G}\left(u_{k}\right)$ is a clique, $u_{k}$ is adjacent to neither $y$ nor $z$. If $u_{k}$ is not adjacent to $x$, then $u_{k}-w-x-y$ is a desired $P_{4}$. Otherwise $u$ is adjacent to $x$ and $u_{k}-x-y-z$ is a desired $P_{4}$.

We are now ready to prove the main theorem in this section.

Theorem 9 Let $G$ be a connected $\left(P_{5}\right.$, paraglider)-free graph. Then $\chi(G) \leq$ $\left\lceil\frac{3 \omega(G)}{2}\right\rceil$. Moreover, $\chi(G)>\frac{3 \omega(G)}{2}$ if and only if $G \in \mathcal{G}$.

Proof. Observe that every graph $H$ in $\mathcal{G}$ has $\chi(H)=8$ and $\omega(H)=5$ and so $\chi(H)=\left\lceil\frac{3 \omega(H)}{2}\right\rceil$. Therefore, if $G \in \mathcal{G}$ then $\chi(G)>\frac{3 \omega(G)}{2}$. We now show by induction on $|V(G)|$ that if $G \notin \mathcal{G}$, then $\chi(G) \leq \frac{3 \omega(G)}{2}$. This will imply the theorem.

First, suppose that $G$ contains a pair of comparable vertices $u$ and $v$, say $N(u) \subseteq N(v)$. Then since $G \notin \mathcal{G}$, it follows that $G-u \notin \mathcal{G}$. Moreover, $G-u$ is connected. By the inductive hypothesis, $\chi(G-u) \leq \frac{3 \omega(G-u)}{2}$. Note that $\chi(G)=\chi(G-u)$ and $\omega(G)=\omega(G-u)$. Therefore, $\chi(G) \leq \frac{3 \omega(G)}{2}$.

Suppose now that $G$ contains a clique cutset. Let $K$ be a minimal clique cutset and $G \backslash K$ is the disjoint union of two subgraphs $H_{1}$ and $H_{2}$. Let $G_{i}=$ $G\left[K \cup V\left(H_{i}\right)\right]$ for $i \in\{1,2\}$. Note that $G_{1}$ and $G_{2}$ are connected. We show that 
neither $G_{1}$ nor $G_{2}$ is in $\mathcal{G}$. Suppose not. We may assume by symmetry that $G_{1} \in \mathcal{G}$. Since $K$ is a non-empty clique of $G_{1}$, it follows from Lemma 6 that there exists an induced $P_{4}:=v-x-y-z$ with $v \in K$ and $x, y, z \notin K$. Since $K$ is minimal, $v$ has a neighbor $w$ in $G_{2}$. Then $w-v-x-y-z$ is a $P_{5}$. This contradicts the fact that $G$ is $P_{5}$-free. Since neither $G_{1}$ nor $G_{2}$ is in $\mathcal{G}, \chi\left(G_{i}\right) \leq \frac{3 \omega\left(G_{i}\right)}{2}$ by the inductive hypothesis. Therefore, $\chi(G)=\max \left\{\chi\left(G_{1}\right), \chi\left(G_{2}\right)\right\} \leq \frac{3 \omega(G)}{2}$.

Suppose that $G$ contains a universal vertex $u$. If $G-u$ is disconnected, then $\{u\}$ is a clique cutset of $G$ and we are done by the argument on clique cutsets. Therefore, $G-u$ is connected. If $G-u \notin \mathcal{G}$, then it follows from the inductive hypothesis that $\chi(G-u) \leq \frac{3 \omega(G-u)}{2}+1=\frac{3(\omega(G)-1)}{2}+1<\frac{3 \omega(G)}{2}$. If $G-u \in \mathcal{G}$, then since every graph in $\mathcal{G}$ has clique number 5 and chromatic number 8 , it follows that $\chi(G)=9$ and $\omega(G)=6$. Thus, $\chi(G)=\frac{3 \omega(G)}{2}$.

Therefore, we may assume that $G$ contains no clique cutsets, universal vertices or pairs of comparable vertices. We now can apply the structure theorem.

If $G$ is an induced subgraph of the complement of the Clebsch graph, then the theorem clearly holds.

If $G$ is a $\overline{P_{3}}$-free expansion of $C_{5}$, then it follows by Lemma 3 that $\chi(G) \leq$ $\left\lceil\frac{5 \omega(G)}{4}\right\rceil \leq \frac{3 \omega(G)}{2}$.

Suppose that $G$ has a good stable set $S$, and that $H_{1}, \ldots, H_{t}$ are the component of $G \backslash S$, where $t \geq 1$. Since $S$ is good, it follows that $\omega\left(H_{i}\right) \leq \omega(G)-1$. We claim that $\chi\left(H_{i}\right) \leq \frac{3}{2} \omega(G)-1$ for each $i \in\{1, \ldots, t\}$. If $H_{i} \notin \mathcal{G}$, then it follow from the inductive hypothesis that $\chi\left(H_{i}\right) \leq \frac{3 \omega\left(H_{i}\right)}{2} \leq \frac{3(\omega(G)-1)}{2}<\frac{3 \omega(G)}{2}-1$. If $H_{i} \in \mathcal{G}$, then $\omega\left(H_{i}\right)=5$ and $\chi\left(H_{i}\right)=8$. This implies that $\omega(G) \geq 6$. Thus, $\chi\left(H_{i}\right)=8=\frac{3}{2} \times 6-1 \leq \frac{3}{2} \omega(G)-1$. Therefore, $\chi(G) \leq \max _{1 \leq i \leq t} \chi\left(H_{i}\right)+1 \leq$ $\frac{3}{2} \omega(G)$.

If $G$ has a stable set $S$ such that $S$ is perfect, then $\chi(G) \leq \chi(G \backslash S)+1=$ $\omega(G \backslash S)+1 \leq \omega(G)+1 \leq \frac{3 \omega(G)}{2}$.

If $G \in \mathcal{H}$, then the theorem follows from Lemma 2 and Theorem 8 .

The following construction shows that our bound in Theorem 9 is tight up to an additive constant. Consider the graph $G_{k}$, for $k \geq 2$ as defined earlier. Note that $G_{k} \in \mathcal{H}$. It is not hard to verify that $G$ is ( $P_{5}$, paraglider)-free, $\alpha\left(G_{k}\right)=2$, and $\omega\left(G_{k}\right)=k+1$. Since $\chi\left(G_{k}\right) \geq \frac{\left|V\left(G_{k}\right)\right|}{\alpha\left(G_{k}\right)}$, we have $\chi\left(G_{k}\right) \geq$ $\frac{3 k}{2}=\frac{3}{2}\left(\omega\left(G_{k}\right)-1\right)$. When $k=2 t+1$ for some integer $t \geq 1$, it follows from Lemma 4 that $\chi\left(G_{k}\right)=3 t+2=\left\lceil\frac{3 \omega\left(G_{k}\right)}{2}\right\rceil-1$. This implies that there is no $\left(\frac{3}{2}-\epsilon\right)$-approximation algorithm for the chromatic number for $\left(P_{5}\right.$, paraglider $)$ free graphs for any $\epsilon>0$.

Theorem 9 has the following two corollaries.

Corollary 1 Every $\left(P_{5}\right.$, paraglider)-free graph $G$ has $\chi(G) \leq\left\lceil\frac{3 \omega(G)}{2}\right\rceil$.

Proof. Let $G_{1}, \ldots, G_{t}$ with $t \geq 1$ be the components of $G$. Then by Theorem 9 , $\chi\left(G_{i}\right) \leq\left\lceil\frac{3 \omega\left(G_{i}\right)}{2}\right\rceil \leq\left\lceil\frac{3 \omega(G)}{2}\right\rceil$. Since $\chi(G)=\max _{1 \leq i \leq t} \chi\left(G_{i}\right)$, the corollary follows.

Corollary 2 Let $G$ be a ( $P_{5}$, paraglider)-free graph (not necessarily connected). Then $\chi(G)>\frac{3 \omega(G)}{2}$ if and only if there exists a component $C$ of $G$ such that $C \in \mathcal{G}$ and $\omega(G) \leq 5$. 
Proof. Clearly, if $G$ has a component $C$ of $G$ such that $C \in \mathcal{G}$ and $\omega(G) \leq 5$, then $\chi(G)=8$ and $\omega(G)=5$ and so $\chi(G)>\frac{3 \omega(G)}{2}$.

Conversely, suppose that $G$ does not satisfy the condition. We show that $\chi(G) \leq \frac{3 \omega(G)}{2}$. Let $G_{1}, \ldots, G_{t}$ with $t \geq 1$ be the components of $G$. If none of $G_{1}, \ldots, G_{t}$ is in $\mathcal{G}$, then by Theorem $9 . \chi\left(G_{i}\right) \leq \frac{3 \omega\left(G_{i}\right)}{2} \leq \frac{3 \omega(G)}{2}$ and so we are done. So we may assume by symmetry that $G_{1} \in \mathcal{G}$. Then $\omega(G) \geq 6$. For each $1 \leq i \leq t$, we show that $\chi\left(G_{i}\right) \leq \frac{3 \omega(G)}{2}$. If $G_{i} \in \mathcal{G}$, then $\chi\left(G_{i}\right)=8=\frac{3}{2} \times 6-1 \leq$ $\frac{3}{2} \omega(G)-1$. If $G_{i} \notin \mathcal{G}$, then $\chi\left(G_{i}\right) \leq \frac{3 \omega(G)}{2}$ by Theorem 9 This completes the proof.

Acknowledgment. We would like to thank Owen Merkel for writing a computer program to verify that the complement of the Clebsch graph and the graph obtained from the complement of the Clebsch graph by deleting a vertex are the only two induced subgraphs of the complement of the Clebsch graph that satisfy $\chi=\left\lceil\frac{3 \omega}{2}\right\rceil$ where the ceiling is necessary, and Lemma 5

\section{References}

[1] A. Brandstädt and C. T. Hoàng. On clique separators, nearly chordal graphs, and the maximum weight stable set problem. Theoretical Computer Science 389 (2007) 295-306.

[2] C. Brause, B. Randerath, I. Schiermeyer and E. Vumar. On the chromatic number of $2 K_{2}$-free graphs. Discrete Applied Mathematics. 253 (2019) 14-24.

[3] S. A. Choudum, T. Karthick, M. A. Shalu. Perfect coloring and linearly $\chi$-bounded $P_{6}$-free graphs. Journal of Graph Theory 54 (2007) 293-306.

[4] S. A. Choudum, T. Karthick, M. A. Shalu. Linear chromatic bounds for a subfamily of $3 K_{1}$-free graphs. Graphs and Combinatorics 24 (2008) 413-428.

[5] M. Chudnovsky, The Erdös-Hajnal conjecture-a survey. Journal of Graph Theory 75 (2014) 178-190.

[6] M. Chudnovsky, T. Karthick, P. Maceli and F. Maffray, Coloring graphs with no induced five-vertex path or gem. Available on: arXiv:1810.06186 [math.CO].

[7] M. Chudnovsky and V.Sivaraman. Perfect divisibility and 2-divisibility. Journal of Graph Theory 90 (2019) 54-60.

[8] M. Chudnovsky, P. Seymour, N. Robertson and R. Thomas. The strong perfect graph theorem. Annals of Mathematics 164 (2006) 51-229.

[9] J. L. Fouquet, V. Giakoumakis, F. Maire and H. Thuillier. On graphs without $P_{5}$ and $\overline{P_{5}}$. Discrete Mathematics 146, 33-44 (1995).

[10] T. Karthick and F. Maffray, Coloring (gem, co-gem)-free graphs. Journal of Graph Theory 89 (2018) 288-303.

[11] T. Karthick and F. Maffray. Vizing bound for the chromatic number on some graph classes. Graphs and Combinatorics 32 (2016) 1447-1460.

[12] H. A. Kierstead and S. G. Penrice. Radius two trees specify $\chi$-bounded classes. Journal of Graph Theory 18 (1994) 119-129.

[13] H. A. Kierstead, S. G. Penrice and W. T. Trotter, On-line and first-fit coloring of graphs that do not induce $P_{5}$. SIAM Journal of Discrete Mathematics 8 (1995) 485-498.

[14] H. A. Kierstead and Y.Zhu. Radius three trees in graphs with large chromatic number. SIAM Journal of Discrete Mathematics 17 (2004) 571-581. 
[15] L. Lovász, A characterization of perfect graphs. Journal of Combinatorial Theory, Series B 13 (1972) 95-98.

[16] A. Gyárfás. Problems from the world surrounding perfect graphs. Zastosowania Matematyki Applicationes Mathematicae 19 (1987) 413-441.

[17] A. Scott and P. Seymour, Induced subgraphs of graphs with large chromatic number. I. Odd holes. Journal of Combinatorial Theory, Series B 121 (2016) 68-84.

[18] I. Schiermeyer and B. Randerath, Polynomial $\chi$-binding functions and forbidden induced subgraphs: A Survey. Graphs and Combinatorics 35 (2019) 1-35. 